\title{
Revisiting Routing Protocols to Design Energy Aware Wireless Body Area Network
}

\author{
N.A. KHAN1, Muhammad Ali1, Hannan Bin Liaqat², Sabit Rahim³, \\ Saleem Ahmed ${ }^{4}$ \\ 1 Electrical Engineering Department, Federal Urdu University of Arts, Science and \\ Technology, Islamabad, Pakistan \\ ${ }^{2}$ Information Technology Department, University of Gujrat, Gujrat, Pakistan \\ ${ }^{3}$ Computer Science Department, Karakorum International University Gilgit- \\ Baltistan, Pakistan \\ ${ }^{4}$ Computer Systems Engineering Department, Dawood University of Engineering \\ and Technology, Karachi, Pakistan \\ E-mail: Naveedali64@yahoo.fr, aliranarana55@gmail.com, \\ hannan.liaqat@uog.edu.pk, sabit.rahim@kiu.edu.pk, saleem3714@gmail.com
}

Received October 30, 2020; Revised December 4, 2020; Accepted January 10, 2021

\begin{abstract}
Wireless body area networks (WBANs) a special type of wireless sensor networks (WSNs) in which sensor nodes to actualize continuous wearable wellbeing observing of patients are able to provide improved healthcare services in a distributed infrastructure less environments. However, the mobile node, due to less battery power, can easily suffer from the problem of energy level when control packets are transfer among nodes-a problem that can occurs by the fact that some sensor nodes may select wrong cluster head with inappropriate path and waste the resources. Although many energy efficient methods have been designed for the traditional sensor networks, there has been limited focus on incorporating WBANs into energy efficient schemes. Therefore, in order to incorporate above issue we revisit the already designed traditional energy efficient methods with cluster head selection protocols and optimal path transformation. Therefore, we encourage researchers to insert WBANs with existing methods to improve performance. However, some work has been done in WBANs that uses energy efficient methods to manage the routing issue, this research domain requires further research attention. Therefore, we discuss the current research work and purpose many future directions of research.
\end{abstract}

Keywords: Wireless body area networks, Energy Aware, Routing Protocols, Stable Increased-throughput, Link Aware-Energy Efficient.

\section{INTRODUCTION}

Wireless sensor networks (WSNs) provide central communication to all nodes that deployed in the specified region of network. However, it has several types that are implemented in the fields of engineering and other 
fields of science. WSNs nodes are useful to measure the temperature, pressure, and humidity. In order to provide communication among nodes medium access control (MAC) layer protocol is used for WSNs and wireless body area networks (WBANs).

WBAN's is specified type of WSNs that is designed to combine multiple data packets from the body and transfer information to central node using WSNs. The lot of research work has been done in the domain of traditional WSNs and many existing surveys provide detail knowledge of already designed protocols. As compare to this, here we are discussing the WSNs protocols that are designed for WBAN. The nodes in WSNs are smaller in size and it has wireless ability with low utilization of power. WBAN is an expected innovation which uses wireless sensor nodes to actualize continuous wearable wellbeing observing of patients. The wearable sensor nodes can be implant inside the body of the patients for the many bio parameters like blood pressure, heart action pulse rate etc. The sensor nodes communicate using radio frequency (RF) based remote systems administration [1]. Patient's wellbeing position can be following whenever and everywhere without confining his/her portability. Consequently, persistent can carry on with his/her life works day by day and exercises.

WSNs have been connected in numerous fields as of late in light of the fact that the nodes of sensors can be conveyed with infrastructure less and system can be monitor numerous perilous and sensor placed that individuals can't reached [2]. Basically low energy adaptive clustering hierarchy (LEACH) is taken under WSNs. In LEACH [3] protocol the base station is not mobile even the area of the nodes is greater to the station. Clustering is proficient strategy to deal with adaptability issue and vitality utilization challenge; it is broadly talked about in WSNs applications [4]. To make WSNs increasingly reasonable for different situations, specialist utilized WSNs with different operating systems [5]. Basically, the heterogeneous WSNs are divided in two major classes: one sensor nodes are conveyed with various correspondence radiuses and other are deployed with various levels of energy [6]. In order to understand the above method this survey mainly focused on LEACH as well as some other WSNs protocols which used for formation the system energy efficient for WBAN.

\section{RELATED WORKS}

WBANs protocols are helpful to minimize the power using of the batteries for maintain the energy efficiency. WSN's is using the minimum level of energy and gives the maximum output with minimum routing overhead [7]. WBANs comprise with little, astute scheme joined on or embedded in the body which are fit for setting up a wireless correspondence interfaces. Sensor nodes are implemented in a person, or around him to handle various applications in the domain of medicine, electronics/personal entertainment 
and others [8]. These devices give ceaseless wellbeing observing and continuous input to the client or medicinal work force. Moreover, the estimations can be recorded over a more drawn out timeframe, improving the nature of the estimated information. The protocols that is helpful in routing plays a vital role for efficient communication among sensor nodes. Furthermore, selection of best path is a major component for communicate with the source and destination [9]. In sensors, nodes send sensory information to the base station that's why communication design is complex.

To avoid challenges in bit rate and energy level, the nodes that have less battery level communicate directly with the access point [10]. Stable increased-throughput multi-hop protocol for link efficiency (SIMPLE), is a low power and energy efficient routing protocol for WBANs. However, the operation of this protocol is consisting of three phases: initial phase, next hope selection and scheduling [11]. In order to reduce energy losses, link aware and energy efficient scheme for body area network (LAEEBA) provides solution after consideration of routing protocol. It provides solution after consideration of best path for transmission. However, the selection criteria of best path are based on the minimum number of hops. Furthermore, the working mechanism of LAEEBA is based on data packet. In order to facilitate emergency data, it uses direct communication with the desired node. However, it uses multi-hop transfer of data when the nature of data is normal [12]. Further extension in LAEEBA, called co-operative link aware and energy efficient scheme for body area network (Co-LAEEBA) is designed for energy efficient WBANs. Co-LAEEBA performed working after utilization of relay nodes; therefore, at a time source node also employed more than one link [13]. iMproved Stable increased-throughput multi-hop protocol for link efficiency (iM-SIMPLE) is an extension of SIMPLE protocol that is also helpful for energy efficient routing protocol in WBANs [14]. In order to perform routing in a cluster manner another approach is available that is called "anybody". Anybody does not make direct communication to the base station because it uses clusters to gather the data. In anybody clusters are chosen stochastically and cluster heads gather the data packet and send this all data packet to define base station [15].

To optimize the network performance using routing method another protocol is available, called BIOCOMM. The working of BIOCOMM in WBANs is based on network layer and MAC layer. Sleeping or hotspot nodes also exist in BIOCOMM but nodes try to finds the path for destination which uses minimum number of hops [16]. Many surveys are available in the domain of WBANs using network layer protocols. However, this survey is focused on the efficiency of energy after consideration of other layers. Therefore, for WBANs some protocols like LEACH LEACH-C [17] BEENISH, PEGASIS and EDDEEC have been discussed in this survey. 


\section{PROPOSED ORIGINALITY: STUDY ON ENERGY AWARE ROUTING PROTOCOL FOR WBANS}

Different energy aware protocols that provide solution using routing method for WBANs are available in Fig. 1. In order to communicate among nodes many WBANs routing protocols are available, that are based on cluster-based routing protocols, temperature based routing mechanism, QoSbased routing method, cross-layered routing protocols, and posturalmovement routing protocol [18].

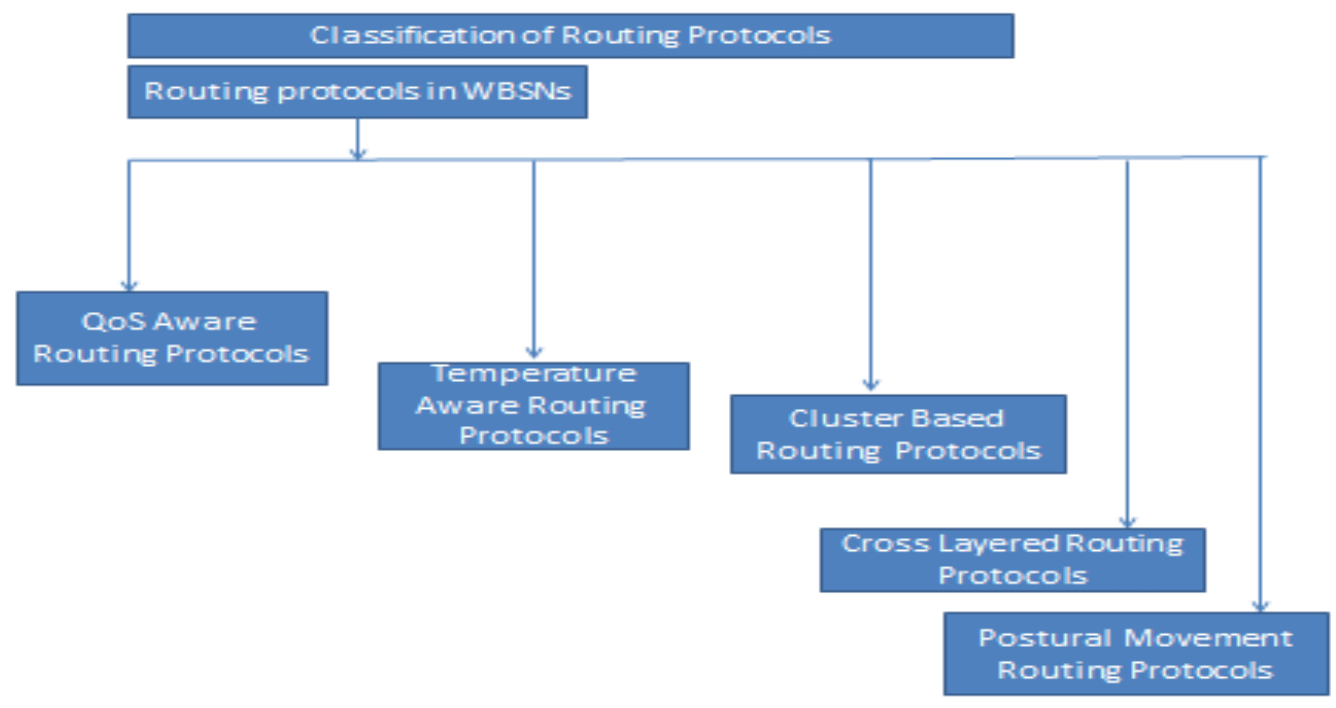

Figure 1. Classification of routing protocol for WBANs [18]

\subsection{Low Energy Adaptive Clustering Protocol (LEACH)}

Basically LEACH is taken under hierarchal network. In LEACH nodes are arrogated to be homogeneous and energy constrained. For homogeneous WSNs, [19-22] discussed about LEACH, that is based on routing protocol in which different group of clusters are used for the sensor network. Sensor network is divided into clusters and in a cluster one node selected that is called cluster heads (CH). CH's have been chosen stochastically in different rounds. Different rounds of LEACH are given.

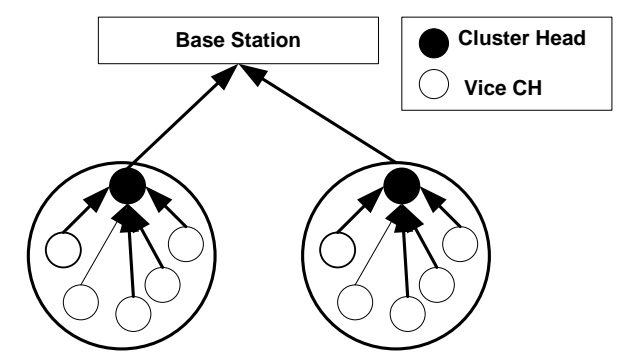

Figure 2. LEACH Protocol Cluster Formation 
Fig. 2 illustrates that all nodes (also called vice cluster head) communicate or sending information to the $\mathrm{CH}$, and then $\mathrm{CH}$ sends information to station which can be stationary. LEACH uses time division multiple access (TDMA) protocol on MAC layer. Furthermore, LEACH divided into different phases. LEACH protocol comprises of numerous rounds and each round comprises of two stages. LEACH protocol is a regularly portrayal of various leveled directing convention and it is self-versatile and selfcomposed [23].

Now the two phases of LEACH are given below.

\subsubsection{Setup Phase}

$\mathrm{CH}$ have been chosen stochastically. During the setup stage, all nodes joined and make the group then select the $\mathrm{CH}$, the node that have maximum energy level is selected as a $\mathrm{CH}$ because it will take longer time to decay [24]. While, the higher energy level node is becoming the $\mathrm{CH}$ then the lower energy level nodes works under main the $\mathrm{CH}$. In this mechanism all subordinate nodes transfer data to $\mathrm{CH}$ that helpful to work under less utilization of energy level resources as sown in Fig.3.

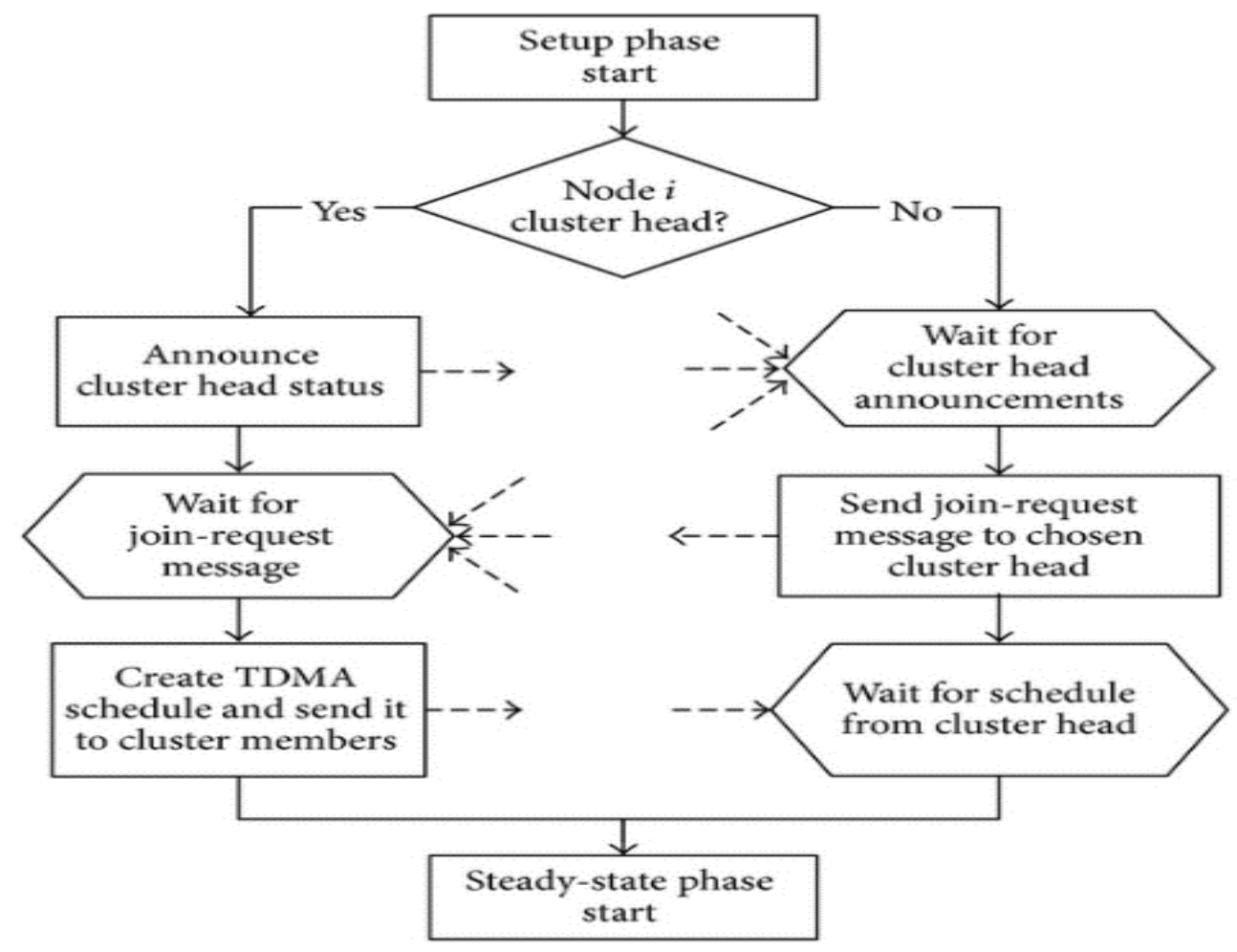

Figure 3. LEACH Setup Phase Flow Chart

\subsubsection{Steady State Phase}

During this phase, all data which is transmitted between nodes is maintained by $\mathrm{CH}$ as illustrated in Fig 4. It combines all data and information 
which came from vice cluster heads and sends this all information directly or through other cluster head to the base station. Moreover, after some specific time interval the whole system returns back to the setup stage [25].

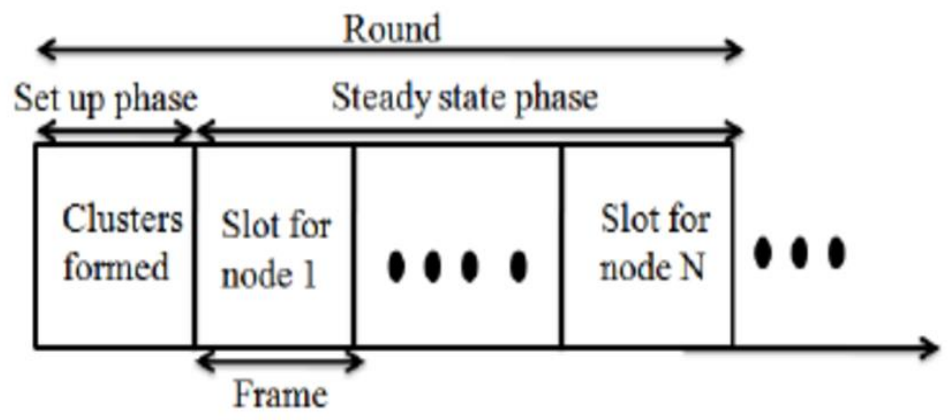

Figure 4. LEACH Steady State Phase

\subsection{Centralized Low Energy Adaptive Clustering Protocol (LEACH-C)}

LEACH-C it is an extension of LEACH, it is uses a centralized clustering approach as mentioned in the name [26]. Base station picked the $\mathrm{CH}$ in LEACH-C then sensor nodes forward their location and energy level to the base station. Group hubs $\mathrm{CH}$ and non- $\mathrm{CH}$ is built up by base station. In this define mechanism as presented in Fig 5, the base station pursues the way that is helpful to achieve the low energy level for transmission of information. In every round of LEACH-C, the quantity of CHs approaches foreordained ideal worth but this is not happening in the case of LEACH.

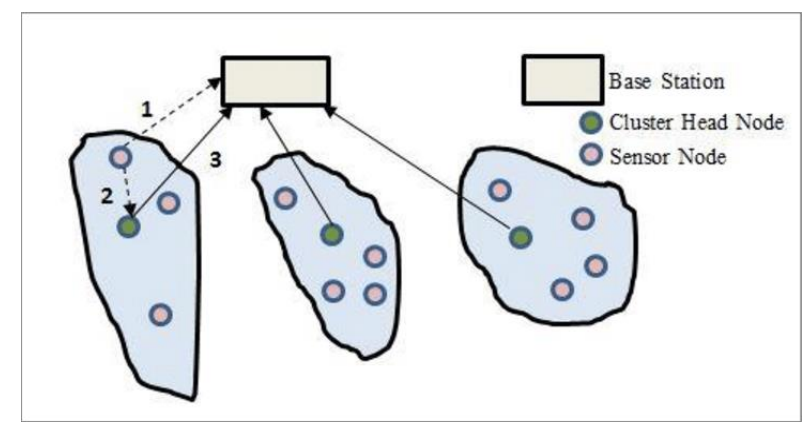

Figure 5. Routing of LEACH-C

\subsection{Balanced Energy Efficient Network Integrated Super Heterogeneous Protocol (BEENISH)}

The main goal in WSNs is to design a framework that should be energy efficient. In order to perform efficiently, a clustering level scheme provides a huge advantage. Therefore, BEENISH protocol used the four dimension of heterogeneity. First one is normal nodes which based on energy level, second one is called propelled nodes which have maximum energy level in order to normal nodes, third one named as super nodes because they have high level of energy, and fourth one is called ultra-super nodes because they have maximum energy level [27]. As per above method, the ultra-super node has 
maximum chance to become the CHs. This is because, it has the highest energy level and as compare to others the duration of life is maximum. As the energy efficiency of BEENISH is higher, therefore, it can send maximum packets to base station. Due to the maximum availability of resources, BEENISH can be very useful for the WBAN.

As Fig. 6 illustrated the block diagram of BEENISH protocol in which all ultrasuper nodes that have greatest energy level are becoming a $\mathrm{CH}$. However, to become the ultra-super node the distance of node does not matter. If ultrasuper node is not present in any area, then the super node becomes the $\mathrm{CH}$. Furthermore, all remaining nodes connect with $\mathrm{CH}$ node and then the $\mathrm{CH}$ connects with base station. Due to the above reasons BEENISH protocol is more effective then LEACHES.

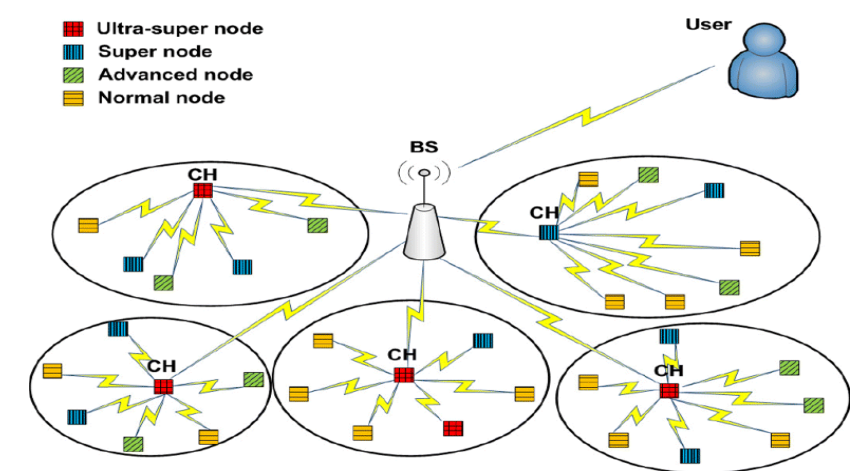

Figure 6. BEENISH Protocol Block Diagram

\subsection{Power Efficient Gathering in Sensor Information System (PEGASIS)}

There are many protocols that are using in WSNs and PEGASIS is one of them. In PEGASIS nodes are composed in string that every node demonstration like transmitter and collector. However, every node can travel and get information from just its neighbor node. $\mathrm{CH}$ is looked over the string which associated with base station and transmits all relevant data to it. The node gets the information from their neighbor node afterward further neighbor collect this information using multi-hop concept. Furthermore, after receiving data from multiple-hops the data transmitted to $\mathrm{CH}$ and finally $\mathrm{CH}$ sends it to the base station. PEGASIS is working with token passing methodology which is utilized by the $\mathrm{CH}$ node from starting to end [28].

As Fig. 7 explain the token passing methodology of PEGASIS. C2 node is $\mathrm{CH}$ which is called pioneer hub and it moves token to $\mathrm{C} 0$ node and $\mathrm{A} 0$ node moves all relevant information to neighbor node $\mathrm{C} 1$. $\mathrm{C} 1$ get together the data and move the accumulated data to $\mathrm{C} 2$. The group head which is called pioneer node, then exchange the token to $\mathrm{C} 4$. The $\mathrm{C} 4$ node move the data to its neighbor node $\mathrm{C} 3$, and $\mathrm{C} 3$ get together the all data and sends data to its neighbor $\mathrm{C} 2$. Toward the end $\mathrm{CH}$ which is $\mathrm{C} 2$ move the total data to base station. Therefore, PEGASIS is increasingly productive then LEACH. 


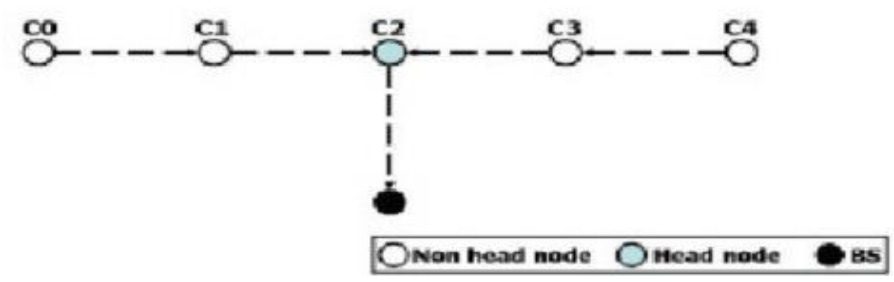

Figure 7. PEGASIS Token Passing Methodology

\subsection{Enhanced Developed Distributed Energy Efficient Clustering (EDDEEC)}

EDDEEC is a versatile energy related protocol that works in a productive manner. Furthermore, it's helpful to provide an equivalent energy level between sensor nodes. In EDDEEC sensor nodes are characterized among three kinds, typical nodes, advance nodes, and super nodes. These three nodes are taken a shot at likelihood manner. On the off chance that energy dimension of ordinary nodes kicks the bucket, the rest of the nodes proceeds with the entire capacity; this may build the energy effectiveness, steadiness and lifetime of the system.

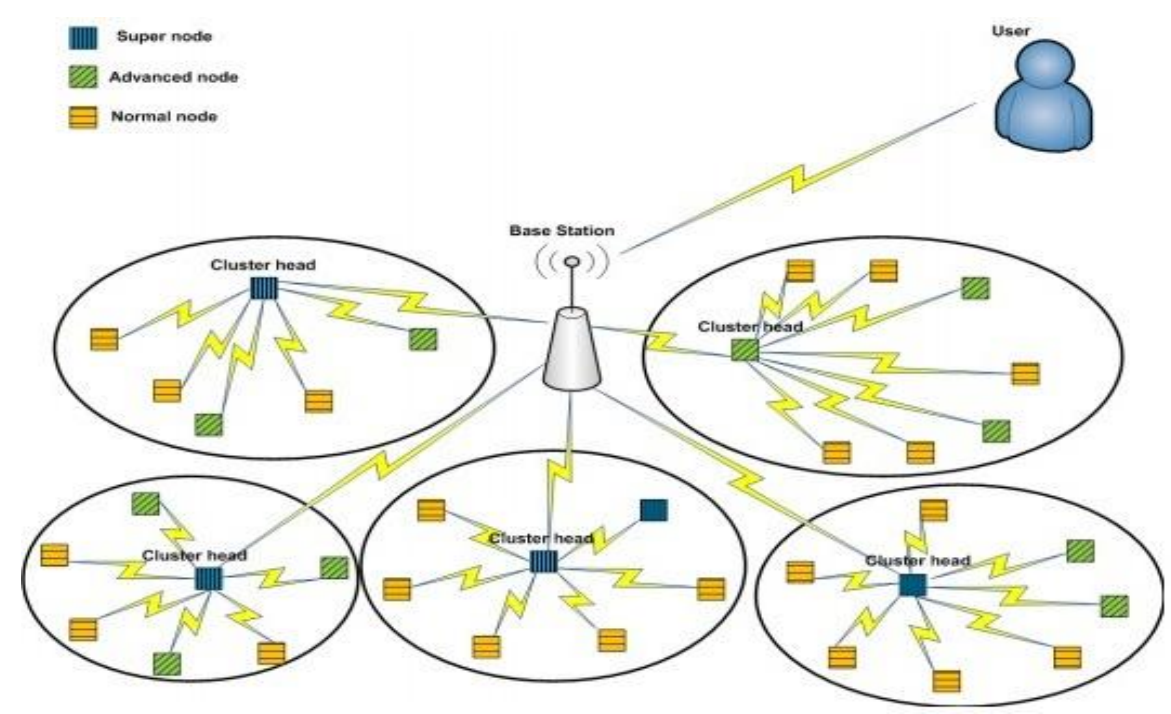

Figure 8. EDDEEC System model

Clustering implicates groups of node's and nominating leaders amid the sensor nodes as shown in Fig . 8. While, the cluster heads are nominated, they collect the data from their corresponding nodes and execute data information accretion afore reportage the gathered data information to the sink node. Rotary the cluster head save majorly energy rather than fixed clustering system. EDDEEC communications protocol ascertains $\mathrm{CH}$ determination solely based on the residuum energy of each node. To achieve this aim, the Energy efficiency EDDEEC is based on following important factors such as: 
- The $\mathrm{CH}$ selection is depends on average distance, initial energy, residual Energy and average energy of the system. While, it is necessary that $\mathrm{CH}$ selection must be purely independent of the subsequent round, un-like the approach in DEEC, SEP, EDDEEC and LEACH.

- The $\mathrm{CH}$ selection totally depends on nodes higher residual energy strength then other nodes in EDDEEC network.

- The EDDEEC must assure equal weightage to normal, super and advance nodes for picking a $\mathrm{CH}$. While others just give the weightage on the bases of advanced and super node.

\section{OBSERVATIONAL RESEARCH ANALYSIS FOR ENERGY EFFICIENT WBANs}

There are many challenges in routing protocol for WBANs. We are discussing some challenges that relates to energy efficiency for WBANs. Energy efficiency of routing protocols is very important and it is the biggest challenge now a day. In energy efficiency both energy consumption of nodes, and network lifetime included and this is the biggest challenge in WBANs[29]. Many algorithms and protocols are available for the energy

TABLE I. COMPARATIVE TABLE OF DifFERENT ENERgy EFFICIENT ROUTING PROTOCOLS

\begin{tabular}{|c|l|l|l|l|c|l|}
\hline $\begin{array}{c}\text { Routing } \\
\text { Protocols }\end{array}$ & Classification & Mobility & $\begin{array}{l}\text { Energy } \\
\text { Efficiency }\end{array}$ & $\begin{array}{l}\text { Power } \\
\text { Usage }\end{array}$ & $\begin{array}{l}\text { Cluster } \\
\text { Head } \\
\text { Energy }\end{array}$ & $\begin{array}{l}\text { For } \\
\text { WBANs }\end{array}$ \\
\hline LEACH & Homogeneous & Fixed & Very poor & Maximum & $\checkmark$ & Good \\
\hline LEACH-C & Homogeneous & Fixed & Very Poor & Maximum & $\checkmark$ & Good \\
\hline PEGASIS & Homogeneous & Fixed & Poor & Minimum & $\checkmark$ & $\begin{array}{l}\text { Very } \\
\text { Good }\end{array}$ \\
\hline BEENISH & Heterogeneous & Fixed & High & Minimum & $\checkmark$ & $\begin{array}{l}\text { Very } \\
\text { Good }\end{array}$ \\
\hline EDDEEC & Heterogeneous & Fixed & High & Average & $\times$ & $\begin{array}{l}\text { Very } \\
\text { Good }\end{array}$ \\
\hline
\end{tabular}

efficiency of nodes. However, still $\mathrm{CH}$ node selection and route selection have greater challenge in WBANs. As we know that WBANs requires quick response of data. Therefore, a proper framework requires for fast response with efficient energy management in WBANs.

Table 1 is describing the energy efficient comparison of LEACH, LEACHC, PEGASIS, BEENISH and EDDEEC for the WBANs. For WBANs, PEGASIS and BEENISH are very good but energy efficiency of BEENISH is higher and energy efficiency of PEGASIS is poor. Remaining LEACH, LEACH-C and 
EDDEEC are good for WBANs but energy efficiency of both are very poor. The efficiency of EDDEEC is higher in terms of energy management.

\section{CONCLUSION}

This survey discussed different energy efficiency of WSNs routing protocols for the WBANs. Some wireless routing protocols like BEENISH and EDDEEC are very good for the energy efficiency of WBANs. BEENISH and EDDEEC are high energy efficient specially BEENISH, less power user and best for body area network. LEACH, LEACH-C and PEGASIS are also good for body area network but its energy efficiency is very poor. In future work the remaining wireless senor protocols can be discussed in WBANs.

\section{REFERENCES}

[1] C. Sevgi and A. Kocyigit, "On determining cluster size of randomly deployed heterogeneous wireless sensor networks," IEEE Communications Letters, vol.12, no.4, pp. 232-234, 2008.

[2] G.Han, H. Xu, T. Q. Duong, J.Jing, and T. Hara, "Localization algorithm of wireless sensor networks: a survey," Telecommunication Systems, vol.52, no.4, pp.2419-2436, 2013.

[3] Del-Valle-Soto, C.; Velázquez, R.; Valdivia, L.J.; Giannoccaro, N.I.; Visconti, P. An Energy Model Using Sleeping Algorithms for Wireless Sensor Networks under Proactive and Reactive Protocols: A Performance Evaluation. Energies 2020, 13, 3024.

[4] S.-S. Wang and Z.-P. Chen, "LCM: a link-aware clustering mechanism for energy-efficient routing in wireless sensor networks," IEEE Sensors Journal, vol. 13, no. 2, pp.728-736, 2013.

[5] T. M. Behera, S. K. Mohapatra, U. C. Samal, M. S. Khan, M. Daneshmand and A. H. Gandomi, "I-SEP: An Improved Routing Protocol for Heterogeneous WSN for IoT-Based Environmental Monitoring," in IEEE Internet of Things Journal, vol. 7, no. 1, pp. 710-717, Jan. 2020, doi: 10.1109/JIOT.2019.2940988.

[6] Mortaza Fahimi Khaton Abad, Mohammad Ali Jabreil Jamali, "Modify LEACH Algorithm for Wireless Sensor Networks," International Journal of Computer science Issues, Vol. 8, Issue 5, No. 1, September 2011.

[7] S. Ullah, H. Higgins, B. Braem, B. Latre, C. Blondia, I. Moerman, S. Saleem, Z. Rahman, and K. Kwak, "A Comprehensive Survey of Wireless Body Area Networks," Journal of Medical Systems, pp. 1-30, 2010.

[8] S. Movassaghi, M. Abolhasan, J. Lipman, D. Smith, and A. Jamalipour, "Wireless body area networks: A survey," IEEE Communications Surveys \&Tutorials, vol. 16, no.3, pp. 1658-1686, 2014. 
[9] A. Kurian and R. Divya, "A survey on energy efficient routing protocols in wireless body area networks (WBAN)," Proc. 2017 Int. Conf. Innov. Information, Embed. Commun. Syst. ICIIECS 2017, vol. 2018Janua, pp. 1-6, 2018.

[10] S. Ullah, H. Higgins, B. Braem, B. Latre, C. Blondia, I. Moerman, S. Saleem, Z. Rahman, and K. S. Kwak, "A comprehensive survey of wireless body area networks," Journal of medical systems, vol. 36, no. 3, pp. 1065-1094, 2012.

[11] Nadeem, N. Javaid, S. Mohammad, M. Khan, S. Sarfraz, and M.Gull, "Simple: Stable increased-throughput multi-hop protocol for link efficiency in wireless body area networks," in Broadband and Wireless Computing, Communication and Applications (BWCCA), 2013 Eighth International Conference on, IEEE, 2013, pp. 221-226.

[12] S. Ahmed, N. Javaid, M. Akbar, A. Iqbal, Z. Khan, and U. Qasim, "Laeeba: Link aware and energy efficient scheme for body area networks," IEEE 28th International Conference on Advanced Information Networking and Applications, IEEE, 2014, pp. 435-440.

[13] S. Ahmed, N. Javaid, S. Yousaf, A. Ahmad, M. Sandhu, M. Imran, Z. Khan, and N. Alrajeh, "Co-laeeba: Cooperative link aware and energy efficient protocol for wireless body area networks," Computers in Human Behaviour, vol. 51, pp. 1205-1215, 2015.

[14] N. Javaid, A. Ahmad, Q. Nadeem, M. Imran, and N. Haider, "iM-SIMPLE: improved stable increased-throughput multi-hop link efficient routing protocol for wireless body area networks," Computers in Human Behavior,vol.51,pp.1003-1011,2015

[15] T. Watteyne, S. Auge-Blum, M. Dohler, and D. Barthel, "Any-body: a self-organization protocol for body area networks," in the Proc. of Second International Conference on Body Area Networks (BODYNETS 2007).

[16] Shahbazi, Z.; Byun, Y.-C. Towards a Secure Thermal-Energy Aware Routing Protocol in Wireless Body Area Network Based on Blockchain Technology. Sensors 2020, 20, 3604.

[17] Qureshi, T.N., Javaid, N., Khan, A.H., Iqbal, A., Akhtar, E. and Ishfaq, M. (2013) BEENISH: Balanced Energy Efficient Network Integrated Super Heterogeneous Protocol for Wireless Sensor Networks. Procedia Computer Science, 19, 920-925.

[18] Qu Y, Zheng G, Ma H, Wang X, Ji B, Wu H. A Survey of Routing Protocols in WBAN for Healthcare Applications. Sensors (Basel). 2019; 19 (7):1638. Published 2019 Apr 5. doi:10.3390/s19071638

[19] Wang, J., Ghosh, R.K. \& Das, S.K. A survey on sensor localization. J. Control Theory Appl. 8, 2-11 https://doi.org/10.1007/s11768-010-9187-7 
[20] M. Z. Hasan, H. Al-Rizzo and F. Al-Turjman, "A Survey on Multipath Routing Protocols for QoS Assurances in Real-Time Wireless Multimedia Sensor Networks," in IEEE Communications Surveys \& Tutorials, vol. 19, no. 3, pp. 1424-1456, thirdquarter 2017, doi: 10.1109/COMST.2017.2661201.

[21] S. Chatterjee and M. Singh, "A centralized energy-efficient routing protocol for wireless sensor networks," Int. J. Adv. Netw. Appl., vol. 3, no. 5, pp. 12-18, 2012.

[22] Singh S.K, Singh M.P and Singh D.K. "A Survey of Energy-Efficient Hierarchical Cluster-Based Routing in Wireless Sensor Networks", International Journal of Advanced Networking and Applications, Vol. 02, Issue 02, pp. 570-580, 2010.

[23] Lalita Yadav, Ch. Sunitha, "Low Energy Adaptive Clustering Hierarchy in Wireless Sensor Network (LEACH)", International Journal of Computer Science and Information Technologies, Vol. 5 (3), 2014.

[24] Reshma I. Tandel "LEACH protocol in wireless sensor network: A survey" International Journal of Computer Science and Information Technologies, Vol. 7(4), 2016, 1894-1896.

[25] Kandris, D.; Nakas, C.; Vomvas, D.; Koulouras, G. Applications of Wireless Sensor Networks: An Up-to-Date Survey.Appl. Syst. Innov. 2020, 3, 14.

[26] A. Kurian and R. Divya, "A survey on energy efficient routing protocols in wireless body area networks (WBAN)," Proc. 2017 Int. Conf. Innov. Information, Embed. Commun. Syst. ICIIECS 2017, vol. 2018Janua, pp. 1-6, 2018.

[27] Aakanksha Sharma, Kamaldeep jangra "A survey- energy efficient routing protocol for homogeneous \& heterogeneous networks" vol 4, issue 5, may -2015 page 328-332.

[28] Singh S.K, Singh M.P and Singh D.K. "A Survey of Energy-Efficient Hierarchical Cluster-Based Routing in Wireless Sensor Networks", International Journal of Advanced Networking and Applications, Vol. 02, Issue 02, pp. 570-580, 2010.

[29] Kandris, D.; Tsioumas, P.; Tzes, A.; Nikolakopoulos, G.; Vergados, D.D. Power conservation through energy efficient routing in wireless sensor networks. Sensors 2015, 9, 7320-7342.

[30] Aidil Saputra Kirsan, Al Rasyid, U. H., Iwan Syarif, \& Dian Neipa Purnamasari. Energy Efficiency Optimization for Intermediate Node Selection Using MhSA-LEACH: Multi-hop Simulated Annealing in Wireless Sensor Network. EMITTER International Journal of Engineering Technology, 8(1), 1-18, 2020. https://doi.org/10.24003/emitter.v8i1.459 